\title{
Diferenças no crescimento e desenvolvimento neuropsicomotor de crianças em centros de educação infantil de Goiânia/GO
}

Difference in growth and neuropsychomotor development in children attending child education centers in Goiânia/GO

Diferencias de crecimiento y desarrollo neuro-psicomotor de niños en centros de educación infantil de Goiânia/GO

Juliana Campos Rodovalho ${ }^{1}$, Ana Karolina Paiva Braga², Cibelle Kayenne Martins Roberto Formiga ${ }^{3}$

\section{RESUMO}

Estudo transversal com objetivos de identificar as diferenças no crescimento e desenvolvimento neuropsicomotor de lactentes e verificar a associação entre os fatores de risco biológicos e ambientais. A amostra foi composta de 45 crianças entre 9 meses a 2 anos de idade que frequentaram creches municipais de Goiânia/GO, em março e abril de 2009. Foram avaliados o crescimento (peso, altura, relação peso/estatura, perímetro cefálico e perímetro torácico) e o desenvolvimento pelo Teste de Denver II, além da coleta de dados socioeconômicos, familiares, gestação, partos e neonatais. As meninas apresentaram maior peso em relação peso/estatura e menor risco de atraso no desenvolvimento pessoal-social. A relação peso/estatura sofreu influência de fatores socioeconômicos e o comprimento foi inversamente proporcional ao risco de atraso no desenvolvimento da linguagem. Reforça-se a importância da promoção de estratégias preventivas de possíveis problemas no desenvolvimento e crescimento das crianças juntamente com pais e cuidadores.

Descritores: Crescimento; Desenvolvimento Infantil; Lactente; Fatores de Risco; Creches.

\section{ABSTRACT}

The objective of this cross-sectional study is to identify the differences in growth and neuropsychomotor development in infants and verify the association between biological and environmental risk factors. The sample consisted of 45 children aged between 9 months and 2 years, who attended municipal daycare centers in Goiânia/GO during the period from March to April 2009. The children were assessed in terms of their growth (weight, height, weight/height ratio, head circumference and chest circumference) and development (using the Denver II Test), in addition to the collection of socioeconomic, family, pregnancy, childbirth, and neonatal data. Girls presented higher weights and height/weight ratios, as well as lower risks for delayed personal-social development. The height/weight ratio was affected by socioeconomic factors and the height was inversely proportional to the risk of delayed language development. The importance of promotion strategies to prevent possible problems in children's development and growth is reinforced among parents and caregivers.

Descriptors: Infant Growth; Child development, infant; Risk Factors; Child Day Care Centers.

\section{RESUMEN}

Estudio transversal que objetivó identificar diferencias en crecimiento y desarrollo neuro-psicomotor de lactantes y verificar asociación entre factores de riesgo biológicos y ambientales. La muestra se constituyó de 45 niños de entre 9 meses y 2 años de edad, que frecuentaban guarderías municipales de Goiânia/GO, de marzo a abril de 2009. Se evaluaron el crecimiento (peso, altura, relación peso/estatura, perímetro cefálico y perímetro torácico) y desarrollo por Test de Denver II, además de colectarse datos socioeconómicos, familiares, del embarazo, parto y neonatales. Las niñas presentaron mayor peso y relación peso/estatura y menor riesgo de atraso en desarrollo personal-social. La relación peso/estatura sufrió influencia de factores socioeconómicos y el cumplimiento fue inversamente proporcional al riesgo de atraso en desarrollo del lenguaje. Se enfatiza la importancia de la promoción de estrategias preventivas de posibles problemas de desarrollo y crecimiento de los niños, conjuntamente con padres y cuidadores.

Descriptores: Crecimiento; Desarrollo Infantil; Lactante; Factores de Riesgo; Jardines Infantiles.

\footnotetext{
${ }^{1}$ Fisioterapeuta. Goiânia, GO, Brasil. E-mail: ju rodovalho@hotmail.com.

${ }^{2}$ Fisioterapeuta. Goiânia, GO, Brasil. E-mail: anakarolinapbraga@hotmail.com.

3 Fisioterapeuta, Doutora em Ciências Médicas, Professora Adjunta, Universidade Estadual de Goiás. Goiânia, GO, Brasil. E-mail: cibellekayenne@gmail.com.
} 


\section{INTRODUÇÃO}

O crescimento e o desenvolvimento são eixos referenciais para todas as atividades de atenção à criança e ao adolescente sob os aspectos biológico, afetivo, psíquico e social(1).

O acompanhamento do crescimento permite avaliar o progresso da criança, identificando aquelas com maior risco de morbimortalidade, prevenindo precocemente a desnutrição e promovendo o crescimento infantil(2). Os desvios do estado nutricional identificados exigem ações imediatas no âmbito da saúde pública, já que além do comprometimento da estatura, várias consequências funcionais adversas estão associadas com a desnutrição(3).

Acompanhar o desenvolvimento da criança nos dois primeiros anos de vida é de fundamental importância, pois é nesta etapa da vida extra-uterina que o organismo mais cresce e amadurece, estando portanto, mais sujeito aos agravos. Devido à grande plasticidade cerebral, nos primeiros anos, a criança responde melhor às terapias e aos estímulos que recebe do meio ambiente(4).

Apesar das variações individuais, o aprendizado dos movimentos se processa em padrões semelhantes e comuns $^{(5)}$. O ser humano nasce com potencial genético de crescimento e desenvolvimento, que poderá ou não ser alcançado, dependendo das condições de vida que lhe sejam propiciadas ${ }^{(6)}$. Nas crianças menores de cinco anos, a influência dos fatores ambientais é muito mais importante do que a dos fatores genéticos para expressão de seu potencial de crescimento. Grande parte das pesquisas realizadas cita a influência do nível socioeconômico (nos aspectos pré, peri e pós-natal) no desenvolvimento neuropsicomotor da criança(1).

Quanto maior o nível de escolaridade da mãe, melhor é a organização do ambiente físico e temporal, há maior oportunidade de variação na estimulação diária, com disponibilidade de materiais e jogos apropriados para a criança e maior envolvimento emocional e verbal da mãe com a criança. Na primeira infância os principais vínculos, bem como os cuidados e estímulos necessários ao crescimento e desenvolvimento, são fornecidos pela família ${ }^{(7)}$

Crianças de baixo nível socioeconômico são frequentemente expostas a múltiplos fatores adversos, constituindo um importante grupo de risco para atrasos no desenvolvimento. Em populações menos favorecidas economicamente, a creche costuma ser uma importante opção de cuidado alternativo para as crianças com idade menor que cinco anos, embora o seu papel em relação ao desenvolvimento infantil permaneça controverso(8).

Alguns estudos relatam que existem diferenças de acordo com o sexo, no brincar infantil, que são importantes na medida em que possibilitam que meninos e meninas desenvolvam-se de maneira diferenciada, adquirindo habilidades diversificadas ${ }^{(9)}$.

Os fatores que influenciam nessa diferenciação podem ser predisposições inatas relacionadas às diferenças entre os gêneros, que são influenciadas pelo ambiente, cultura ou múltiplos fatores que interagem ao longo do processo de desenvolvimento(9).

No Brasil, a avaliação do crescimento desde o nascimento até 72 meses é feita através dos gráficos Estatura/Idade e Peso/Idade, que ilustram o crescimento linear e sua velocidade, respectivamente. As curvas utilizadas pelo Ministério da Saúde, presentes na caderneta da criança, são curvas do National Center for Health Statistics (NCHS), adotado pela Organização Mundial da Saúde (OMS) como padrão internacional(1).

Por não se ter no Brasil um instrumento padronizado para avaliação do desenvolvimento, há dificuldade na identificação de atrasos. Em geral são aplicados testes que avaliam setores específicos do desenvolvimento, sendo necessária a realização de dois ou mais testes para obter a avaliação global do desenvolvimento. Isso contribui para que alterações no desenvolvimento passem despercebidas, só se tornando evidentes muito tarde, quando a criança ingressa no ensino fundamental(10). Deste modo, a identificação precoce de crianças com atrasos no desenvolvimento motor é um desafio para os profissionais da saúde.

Portanto, os objetivos do presente estudo foram, identificar as diferenças no crescimento e desenvolvimento neuropsicomotor de lactentes e verificar a associação entre os fatores de risco biológicos e ambientais.

\section{METODOLOGIA}

A pesquisa teve o caráter transversal e contou com uma amostra de conveniência de 45 lactentes, com idade entre nove meses a dois anos de idade, que frequentaram as creches municipais envolvidas e atenderam aos critérios de inclusão. As avaliações foram feitas nos meses de março e abril de 2009. 
Para participar da pesquisa, as crianças deveriam ter até 2 anos, estar cadastradas nas instituições que aderiram ao projeto e ser autorizadas pelos pais ou responsável, por escrito. Os critérios para exclusão dos participantes foram: gestações de alto risco, prematuridade, baixo peso ao nascimento, retardo do crescimento intra-uterino, presença de alguma patologia neonatal que levasse à necessidade de tratamento intensivo no período neonatal, asfixia perinatal, diagnóstico de infecções congênitas, anomalias cromossômicas ou malformações graves diagnosticadas.

Este estudo foi realizado em cinco CMEls (Centros Municipais de Educação Infantil) da cidade de Goiânia/GO, pertencentes a diferentes regiões da cidade, considerando setores com valorização imobiliária distintos, que aderiram ao estudo e atendiam crianças na faixa etária estabelecida. A elaboração da pesquisa foi baseada nas Diretrizes e Normas Regulamentadoras de Pesquisas Envolvendo Seres Humanos (Resolução 196/1996, do Conselho Nacional de Saúde). O projeto foi submetido e aprovado pelo Comitê de Ética em Pesquisa da Universidade Federal de Goiás, por meio do protocolo de número 004/2009 e pela Secretaria Municipal da Educação de Goiânia/GO.

Os materiais utilizados na avaliação do crescimento dos lactentes foram: fita métrica para avaliação do perímetro cefálico e torácico; antropômetro horizontal para registro do comprimento; balança digital para avaliação do peso; as curvas de crescimento do NCHS National Center for Health Statistics, que utilizam as estaturas médias em centímetros (cm), atingidas nas sucessivas idades para a população de referência, de acordo com a OMS, e permite a construção de índices antropométricos ${ }^{(11)}$.

$\mathrm{Na}$ avaliação do desenvolvimento neuropsicomotor foi utilizado o Teste de Triagem de Denver II adaptado para o português, que consiste em 125 itens distribuídos em quatro áreas do desenvolvimento: pessoal-social, motor fino, linguagem e motor grosseiro ${ }^{(12)}$. Os materiais utilizados no teste foram: um pompom vermelho de lã, uvas passas, um chocalho com cabo estreito, 10 cubos coloridos de madeira, um pequeno pote transparente, um sino pequeno, uma bola de tênis, um lápis vermelho, uma boneca de plástico com uma mamadeira, uma caneca plástica com asa, e papel branco.

Para registrar o contexto de saúde e condições de vida da criança foram utilizados: roteiro de dados da criança, onde constaram informações da criança e dos pais (idade, escolaridade, profissão, endereço), dados referentes à gestação (número de gestações, abortos, consultas no pré-natal, patologias na gravidez, peso adquirido na gestação, hábitos de vida da mãe e idade gestacional), ao parto (tipo de parto, complicações durante o mesmo e local) e dados neonatais contidos na caderneta da criança (Apgar, peso, comprimento, perímetro cefálico e torácico ao nascimento), bem como a presença de patologias diagnosticadas, tempo de internação, tempo de aleitamento materno e aleitamento exclusivo e o protocolo do questionário socioeconômico da ABEP (Associação Brasileira de Empresas de Pesquisa) ${ }^{(13)}$.

Os pais foram convidados a participar da pesquisa e foram esclarecidos sobre o objetivo da mesma e do caráter voluntário de participação. Quando houve concordância, os pais e/ou responsável preencheram e assinaram o Termo de Consentimento Livre e Esclarecido. Apenas um responsável presente no momento dos esclarecimentos negou a participação. Em seguida foram coletadas junto aos responsáveis, as informações do roteiro de dados da criança e do questionário da ABEP.

Com os lactentes incluídos, foram feitas as avaliações antropométricas e do desenvolvimento neuropsicomotor segundo o Teste de Denver II.

Durante o Teste de Triagem de Denver II, a criança foi estimulada a executar atividades consideradas próprias para a sua idade. Рara classificação foram considerados casos indicativos de suspeita de atraso no desenvolvimento aqueles em que a criança apresentou dois ou mais itens de "cuidados", isto é, a criança não realiza a tarefa quando $75-90 \%$ das crianças da faixa etária o realiza, e/ou um ou mais itens de "atrasos", ou seja, não realização do item quando $90 \%$ ou mais das crianças o realiza. Na presença de dois cuidados ou um atraso, a criança foi classificada como risco ${ }^{(14)}$.

A análise dos dados foi feita de acordo com o roteiro de dados da criança, questionário socioeconômico aplicado, avaliações antropométricas e resultados obtidos no Teste de Denver II.

O Banco de Dados foi preparado no programa Microsoft Office Excel. Os dados relacionados à classificação foram apresentados de acordo com códigos, estipulados pela pesquisadora. 
A análise estatística foi realizada em etapas. Na primeira etapa realizou-se a análise descritiva dos dados, incluindo cálculo de médias, desvio padrão, frequências e porcentagens. Posteriormente foi realizado o teste de correlação de Spearman entre os fatores de risco biológicos e ambientais e entre os itens de avaliação do crescimento e desenvolvimento. Foi utilizado o teste TStudent para comparação de grupos independentes (sexo masculino $x$ sexo feminino) quanto às variáveis biológicas, ambientais e de crescimento. Por fim, foi utilizado o teste Qui-Quadrado para a comparação de grupos quanto ao sexo em relação às variáveis categóricas de crescimento (classificação do peso, altura e relação peso/estatura) e desenvolvimento (risco $x$ normal). Em todas as análises foi considerado o nível de significância estatística de $5 \%(p \leq 0,05)$.

\section{RESULTADOS}

A amostra foi composta por 45 crianças com idade entre nove meses a dois anos, frequentadores de cinco CMEls de diferentes regiões da cidade de Goiânia/GO, número equivalente a $5 \%$ do total de CMEls da cidade; $65 \%$ das crianças eram do sexo feminino e $96 \%$ fizeram uso de aleitamento materno e o tempo de aleitamento exclusivo teve média de quatro meses.

Através dos dados coletados, observou-se que a maioria das famílias pertencia à classe econômica $C$ (51\%). A maior parte das mães (53\%) e dos pais (54\%) possuía o ensino médio completo e aproximadamente $84 \%$ das mães possuíam emprego. Durante a gestação, $98 \%$ das mães fizeram o pré-natal, $71 \%$ dos partos foram cesáreas e $66 \%$ deles em hospital público.

Os dados obtidos em avaliações e outras variáveis foram comparados entre meninos e meninas. Os resultados da comparação das características e crescimento das crianças de acordo com o sexo estão descritos na Tabela 1. 
Tabela 1: Resultados da comparação das características e crescimento das crianças de acordo com o sexo. Goiânia, GO, 2009.

\begin{tabular}{|c|c|c|c|}
\hline Variável & Sexo & Média & Valor de $\mathbf{p}$ \\
\hline \multirow{2}{*}{$\mathrm{N}^{\circ}$ de consultas no pré-natal } & Feminino & 9,07 & \multirow{2}{*}{0,306} \\
\hline & Masculino & 9,87 & \\
\hline \multirow{2}{*}{ Idade gestacional (semanas) } & Feminino & 38,93 & \multirow{2}{*}{0,175} \\
\hline & Masculino & 39,44 & \\
\hline \multirow{2}{*}{ Idade da mãe (anos) } & Feminino & 27,59 & \multirow{2}{*}{0,769} \\
\hline & Masculino & 28,19 & \\
\hline \multirow{2}{*}{ Idade do pai (anos) } & Feminino & 31,04 & \multirow{2}{*}{0,445} \\
\hline & Masculino & 33,94 & \\
\hline \multirow{2}{*}{ Peso ao nascer (gramas) } & Feminino & 3162,59 & \multirow{2}{*}{0,379} \\
\hline & Masculino & 3279,06 & \\
\hline \multirow{2}{*}{ Comprimento ao nascer $(\mathrm{cm})$} & Feminino & 49,59 & \multirow{2}{*}{0,836} \\
\hline & Masculino & 49,44 & \\
\hline \multirow{2}{*}{ Perímetro cefálico ao nascer $(\mathrm{cm})$} & Feminino & 34,04 & \multirow{2}{*}{$0,002^{*}$} \\
\hline & Masculino & 36,40 & \\
\hline \multirow{2}{*}{ Apgar no $1^{\circ}$ minuto } & Feminino & 8,21 & \multirow{2}{*}{0,734} \\
\hline & Masculino & 8,38 & \\
\hline \multirow{2}{*}{ Apgar no $5^{\circ}$ minuto } & Feminino & 9,64 & \multirow{2}{*}{0,646} \\
\hline & Masculino & 9,54 & \\
\hline \multirow{2}{*}{ Tempo de aleitamento materno (meses) } & Feminino & 7,90 & \multirow{2}{*}{0,239} \\
\hline & Masculino & 10,13 & \\
\hline \multirow{2}{*}{ Tempo de aleitamento exclusivo (meses) } & Feminino & 3,79 & \multirow{2}{*}{0,342} \\
\hline & Masculino & 4,38 & \\
\hline \multirow{2}{*}{ Renda familiar (reais) } & Feminino & 891,00 & \multirow{2}{*}{0,578} \\
\hline & Masculino & 985,94 & \\
\hline \multirow{2}{*}{ Peso na avaliação (gramas) } & Feminino & 10972,41 & \multirow{2}{*}{0,210} \\
\hline & Masculino & 10312,50 & \\
\hline \multirow{2}{*}{ Comprimento na avaliação (cm) } & Feminino & 76,84 & \multirow{2}{*}{0,334} \\
\hline & Masculino & 80,063 & \\
\hline \multirow{2}{*}{ Perímetro cefálico na avaliação (cm) } & Feminino & 46,98 & \multirow{2}{*}{0,192} \\
\hline & Masculino & 47,62 & \\
\hline \multirow{2}{*}{ Perímetro torácico na avaliação (cm) } & Feminino & 49,69 & 0327 \\
\hline & Masculino & 48,91 & ו ו \\
\hline
\end{tabular}

$* p<0.05$

Dentre as características das crianças apenas o perímetro cefálico apresentou diferença estatisticamente significativa entre os sexos, indicando que as crianças do sexo masculino nasceram com maior perímetro cefálico que as do sexo feminino.

A Tabela 2 mostra a comparação entre os sexos em relação à avaliação do crescimento.

Tabela 2: Resultados da avaliação do crescimento de acordo com o sexo. Goiânia, GO, 2009.

\begin{tabular}{|c|c|c|c|c|c|c|c|c|c|}
\hline \multirow{2}{*}{ Sexo } & \multicolumn{3}{|c|}{ Peso } & \multicolumn{3}{|c|}{ Estatura } & \multicolumn{3}{|c|}{ Relação peso/estatura } \\
\hline & Baixo & Normal & Sobrepeso & Baixa & Média & Alta & Desnutrição & Normal & Sobrepeso \\
\hline Feminino & $1(3 \%)$ & $24(83 \%)$ & $4(14 \%)$ & 0 & $28(97 \%)$ & $1(3 \%)$ & 0 & $27(93 \%)$ & $2(7 \%)$ \\
\hline Masculino & $5(31 \%)$ & $11(69 \%)$ & 0 & 0 & $16(100 \%)$ & 0 & $3(19 \%)$ & $13(81 \%)$ & 0 \\
\hline Valor de p & & 0,004 & & & 0,644 & & & 0,014 & \\
\hline
\end{tabular}

Pode-se observar que a maior parte das crianças com peso abaixo da linha de normalidade era de sexo masculino e a maioria das classificadas como normais ou com sobrepeso era do sexo feminino, com diferenças estatisticamente significativas. 
Quanto à classificação da altura foram classificadas como altas apenas meninas (3\%), como de média estatura, $100 \%$ dos meninos e $97 \%$ das meninas.

A comparação da relação peso/estatura de acordo com o sexo revelou que $19 \%$ dos meninos estavam desnutridos e $7 \%$ das meninas com sobrepeso.
A comparação do desenvolvimento neuropsicomotor das crianças de acordo com o sexo encontra-se ilustrada no Gráfico 1.

Gráfico 1: Prevalência de risco no desenvolvimento de acordo com o sexo. Goiânia, GO, 2009.

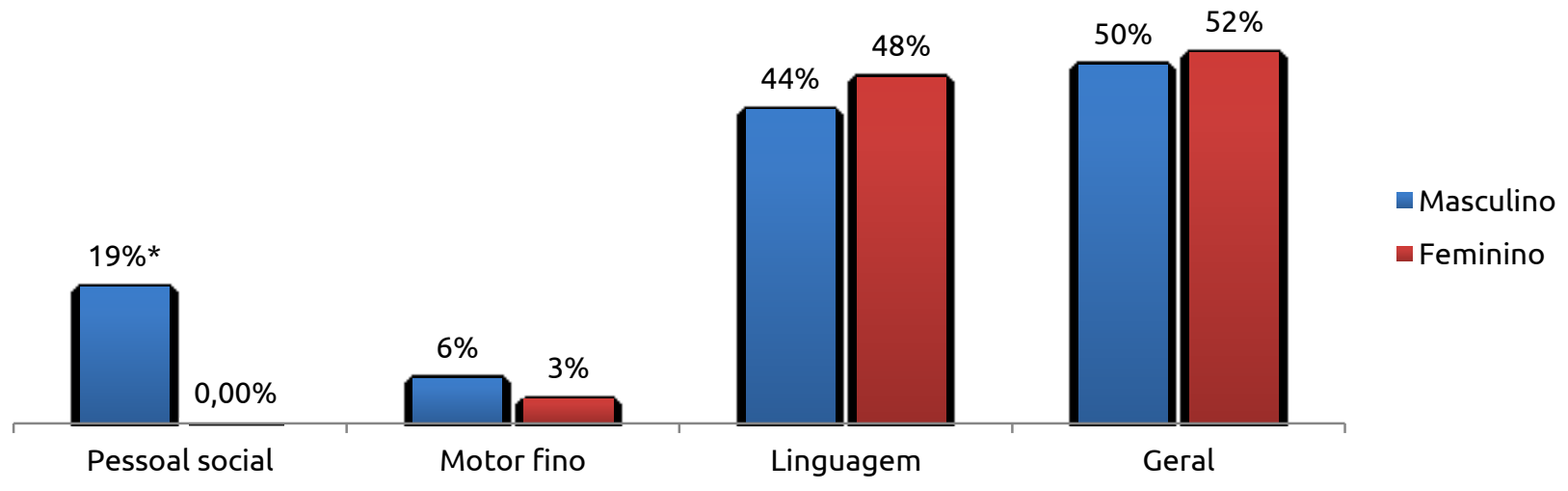

Teste Qui-Quadrado. * Pessoal Social com $\mathrm{p}=0,04$.

Comparando desenvolvimento

neuropsicomotor das crianças de acordo com o sexo, observa-se que as crianças do sexo masculino apresentaram maior risco no desenvolvimento pessoalsocial. Nas demais áreas não houve diferença estatisticamente significativa.

Através da correlação entre as características biológicas e ambientais e os dados colhidos quanto ao crescimento e o desenvolvimento das crianças, observou-se a associação estatisticamente significativa entre algumas variáveis. Essas associações estão descritas na Tabela 3. 
Tabela 3: Resultados da associação entre variáveis ambientais e biológicas. Goiânia, GO, 2009.

\begin{tabular}{|c|c|c|c|}
\hline Variável 1 & Variável 2 & Coef. Correlação (r) & Valor de $\mathrm{p}$ \\
\hline \multirow{5}{*}{ Apgar no $5^{\circ}$ minuto } & Peso ao nascer & 0,374 & 0,016 \\
\hline & Renda familiar & 0,375 & 0,016 \\
\hline & Apgar no $5^{\circ}$ minuto & 0,39 & 0,012 \\
\hline & Número de gestações da mãe & $-0,33$ & 0,029 \\
\hline & Número de partos da mãe & $-0,478$ & 0,001 \\
\hline \multirow[t]{7}{*}{ Classe econômica } & Número de consultas no pré-natal & 0,334 & 0,027 \\
\hline & Número de irmãos & $-0,311$ & 0,038 \\
\hline & Tempo de aleitamento exclusivo & 0,364 & 0,014 \\
\hline & Número de consultas no pré-natal & 0,334 & 0,027 \\
\hline & CMEl onde a criança frequenta & 0,297 & 0,048 \\
\hline & Número de gestações da mãe & $-0,458$ & 0,002 \\
\hline & Número de partos da mãe & $-0,603$ & $<0,001$ \\
\hline \multirow{5}{*}{ Escolaridade da mãe } & Escolaridade do pai & 0,377 & 0,018 \\
\hline & Tipo de parto & 0,526 & $<0,001$ \\
\hline & Local do parto & 0,341 & 0,024 \\
\hline & Classe econômica & 0,745 & $<0,001$ \\
\hline & Renda familiar & 0,671 & $<0,001$ \\
\hline \multirow{3}{*}{ Escolaridade do pai } & Peso ganho na gravidez & 0,616 & $<0,001$ \\
\hline & Local do parto & 0,393 & 0,013 \\
\hline & Número de partos da mãe & $-0,368$ & 0,014 \\
\hline \multirow{3}{*}{ Renda familiar } & Quantidade de patologias na gravidez & $-0,312$ & 0,039 \\
\hline & Escolaridade do pai & 0,36 & 0,025 \\
\hline & Tipo de parto & 0,308 & 0,039 \\
\hline Se a mãe tem emprego ou não & Quantidade de patologias na gravidez & $-0,304$ & 0,045 \\
\hline \multirow{2}{*}{ Sexo da criança } & Perímetro cefálico ao nascer & 0,566 & $<0,001$ \\
\hline & Horas de internação pós-nascimento & $-0,332$ & 0,026 \\
\hline Tipo de parto & Número de partos da mãe & $-0,4$ & 0,007 \\
\hline
\end{tabular}

DP: desvio padrão; f: frequência

A classificação econômica foi diretamente proporcional ao número de consultas no pré-natal, ao Apgar no $5^{\circ}$ minuto e ao tempo de aleitamento exclusivo. Foi inversamente proporcional ao número de gestações da mãe. Maiores valores de renda familiar estiveram relacionados com o tipo de parto cesárea, menor número de partos e de patologias na gravidez, melhor Apgar no $5^{\circ}$ minuto e maior escolaridade dos pais.

As mães que trabalhavam fora do lar apresentaram menor número de patologias na gravidez. A maior escolaridade da mãe se relacionou com um menor número de gestações, maior aumento de peso na gestação, maior classe econômica, parto em hospital privado e do tipo cesárea.
Os resultados da associação entre variáveis do desenvolvimento, crescimento e ambientais estão dispostos na Tabela 4. 
Tabela 4: Resultados da associação entre variáveis do desenvolvimento, crescimento e ambientais. Goiânia, GO, 2009.

\begin{tabular}{|c|c|c|c|}
\hline Variável 1 & Variável 2 & Coef. Correlação (r) & Valor de $p$ \\
\hline & Comprimento & $-0,364$ & 0,014 \\
\hline \multirow[t]{2}{*}{ Risco de atraso na Linguagem } & Risco de atraso Geral & 0,915 & $<0,001$ \\
\hline & Apgar no $5^{\circ}$ minuto & $-0,362$ & 0,02 \\
\hline Risco de atraso no Pessoal social & Sexo da criança & 0,36 & 0,015 \\
\hline \multirow[t]{2}{*}{ Risco de atraso Geral } & Apgar no $5^{\circ}$ minuto & $-0,398$ & 0,01 \\
\hline & Sexo da criança & $-0,37$ & 0,012 \\
\hline \multirow[t]{2}{*}{ Relação Peso/estatura } & Se a mãe tem emprego ou não & 0,342 & 0,022 \\
\hline & CMEl onde a criança frequenta & 0,328 & 0,028 \\
\hline \multirow{2}{*}{ Classificação do peso } & Sexo da criança & $-0,428$ & 0,003 \\
\hline & Tipo de parto & 0,348 & 0,019 \\
\hline Percentil de perímetro cefálico & CMEl onde a criança frequenta & $-0,339$ & 0,023 \\
\hline
\end{tabular}

As crianças de sexo feminino apresentaram menor perímetro cefálico ao nascer e maior tempo de internação pós-nascimento.

De acordo com a correlação, os fatores de desenvolvimento, crescimento e ambientais pode-se observar que a relação peso/estatura foi maior em crianças que frequentavam creches localizadas em setores mais nobres da cidade de Goiânia, onde as mães possuíam um maior nível de escolaridade e trabalhavam. Crianças nascidas por parto cesárea obtiveram maior peso na avaliação, quando comparadas àquelas nascidas por parto normal.

O risco de atraso na linguagem correlacionou-se com menor comprimento e com o risco de atraso geral do Teste de Denver II. Já o Apgar no $5^{\circ}$ minuto foi inversamente proporcional ao risco de atraso na linguagem e geral.

As crianças de sexo feminino apresentaram menor risco de atraso no desenvolvimento pessoal-social, maior peso e relação peso/estatura na avaliação.

\section{DISCUSSÃO}

Os resultados apresentados permitem observar que enquanto a maior parte das crianças com peso abaixo da linha de normalidade era de sexo masculino e a maioria das classificadas como normais ou com sobrepeso era do sexo feminino, o que se assemelha em partes a outro estudo realizado em Catanduva/SP, em que das 113 crianças estudadas, a obesidade foi mais frequente no sexo feminino (61\%). Porém não houve diferença de prevalência de desnutrição entre os sexos ${ }^{(12)}$.

De uma forma geral, a maioria das crianças encontrava-se com peso e estatura dentro da faixa de normalidade para a sua idade. Esses resultados podem ser justificados pelo fato de que a maioria das crianças fez uso de aleitamento materno e permaneceram em média quatro meses em aleitamento exclusivo.

Além do aleitamento materno, talvez o fato de que todas as unidades dos CMEls apresentam cardápio aprovado por nutricionistas contratados pela Secretaria Municipal de Educação e de que as crianças permanecem 19 horas nas instituições e recebem cinco refeições diárias, podem estar relacionado aos resultados encontrados.

Quanto ao desenvolvimento neuropsicomotor, o presente estudo verificou que as crianças de sexo feminino apresentaram risco de atraso ligeiramente maior que as do sexo masculino na área da linguagem e, consequentemente, na avaliação geral do desenvolvimento. Este resultado foi diferente do estudo patrocinado pela OMS, que utilizou o teste de Dever II para avaliar a linguagem, onde a concentração de suspeita de atraso de linguagem de acordo com o sexo foi de $58 \%$ para os meninos e $41 \%$ para as meninas ${ }^{(15)}$.

$\mathrm{Na}$ pesquisa realizada em Canoas/RS com 197 crianças de zero a seis anos, das 53 crianças que apresentaram suspeita de atraso no desenvolvimento, $60 \%$ eram do sexo masculino e $40 \%$ do sexo feminino ${ }^{(16)}$.

Provavelmente o predomínio de atraso na linguagem se justifica pela impossibilidade de uma devida estimulação tanto pelas cuidadoras, no período em que as crianças estão nas creches, quanto pelos pais no momento em que se reúnem em casa. A linguagem também apresenta uma particularidade, pois é uma das áreas do desenvolvimento que se desenvolve através da 
interação com outras pessoas, ou seja, a criança não se desenvolve sozinha nesta área.

Os autores do estudo realizado em creches públicas de Recife/PE, que investigaram os fatores associados ao desenvolvimento mental e motor, afirmaram que houve uma grande concentração de crianças nas salas, contrastando com um número restrito de educadoras. Essa sobrecarga de trabalho faz com que as educadoras passem a se ocupar mais com os cuidados básicos de higiene e alimentação. Esse fato, somado a uma carência de conhecimentos sobre técnicas de estimulação do desenvolvimento infantil, pode comprometer a qualidade da estimulação(8).

Em alguns aspectos, os resultados obtidos se aproximam dos resultados citados em um estudo realizado em uma creche municipal da periferia de Guarulhos (SP), onde foi avaliado o crescimento e desenvolvimento, pelo teste de Denver II, de 44 crianças entre 12 e 36 meses e constatado que as crianças classificadas como questionáveis apresentaram atrasos nas áreas da linguagem (84\%) ${ }^{(6)}$.

Uma pesquisa avaliou 204 crianças em Canoas/RS com prevalência de suspeita de atraso no desenvolvimento neuropsicomotor de $27,5 \%$, sendo que a prevalência de suspeita de atraso de linguagem foi de $26 \%$. Os autores afirmam que as situações estressantes vividas por crianças de baixa renda e criadas por mães com baixa escolaridade, afetam sobremaneira a estrutura familiar e o estímulo à linguagem infantil, comprometendo a aquisição e o desenvolvimento da linguagem ${ }^{(16)}$

O fato do atraso de linguagem estar presente no período anterior ao ingresso na escola, não significa que esta alteração não será suprida pela escola, nem que a criança desenvolverá sérios problemas de aprendizado. Durante os primeiros anos de vida a criança adquire conhecimentos através de práticas, ações, habilidades e comportamentos que deverão acompanhá-la durante sua vida adulta, e influenciar seus valores culturais e sua interação com as pessoas no campo pessoal e profissional ${ }^{(15)}$.

As crianças do sexo feminino apresentaram menor risco de atraso no desenvolvimento pessoal-social, o que está de acordo com a afirmação de que, desde a creche, as meninas desenvolvem mais do que os meninos as suas capacidades de interação e mostram uma maior capacidade de contato social, principalmente quando estão em dupla(17).

Quanto ao desenvolvimento motor grosseiro, não houve nenhuma suspeita de atraso, provavelmente devido às condições oferecidas na creche, opostas às de um estudo em que foram analisadas as oportunidades para o desenvolvimento motor em ambientes domésticos de diferentes níveis socioeconômicos no Ceará. Foi avaliado o espaço físico interno e externo, aparatos internos e externos, superfícies internas e espaços internos para brincadeiras existentes no lar. Verificou-se que, independentemente do nível econômico o espaço exterior da maioria das residências analisadas não oferecia oportunidades suficientes para o desenvolvimento motor das crianças e por isso foram sugeridos investimentos na criação de creches e espaços públicos e contratação de profissionais capacitados para atender a essas necessidades ${ }^{(18)}$.

Já em outra pesquisa foi avaliado o desenvolvimento cognitivo de crianças frequentando creche, em comparação com crianças que permaneciam em casa, todas de um bairro pobre de Salvador e com um mesmo nível socioeconômico. Os resultados mostraram que o desenvolvimento cognitivo foi afetado pela renda das famílias e, parcialmente, pela escolaridade materna, mas que frequentar a creche não produziu melhora no desenvolvimento(4).

No presente estudo, pode-se observar que a maior parte das famílias pertencia à classe econômica C (51\%). Maiores valores de renda familiar relacionaram-se com o parto cesárea, menor número de partos e de patologias na gravidez. As mães com maior classificação econômica segundo os critérios da ABEP fizeram maior número de consultas no pré-natal, seus filhos obtiveram maior Apgar no $5^{\circ}$ minuto e tiveram maior tempo de aleitamento exclusivo.

Em uma pesquisa em que $34 \%$ das crianças avaliadas apresentaram suspeita de atraso no desenvolvimento pelo teste de Denver II, as crianças que tinham maior risco de suspeita de atraso no desenvolvimento foram as de classes sociais menos favorecidas, as que haviam nascido com menor peso, as que apresentaram idade gestacional menor do que 37 semanas, as que tinham mais de três irmãos e as que haviam recebido leite materno por menos de três meses, ou não haviam sido amamentadas $^{(14)}$. 
No atual estudo, a maior parte das mães e dos pais possuía o ensino médio completo e a maioria das mães possuía trabalho remunerado, e esse fato representou um menor número de patologias na gravidez. Aquelas mães com maior nível de escolaridade tiveram um menor número de gestações, maior aumento de peso na gestação, fizeram o parto em hospital privado e do tipo cesárea. Esses achados são coerentes com a afirmação de que a entrada maciça de mulheres casadas e com filhos no mercado de trabalho nas últimas décadas levaram à queda da taxa de fecundidade, à redução do tamanho das famílias e ao aumento do número de famílias chefiadas por mulheres devido à atualização ideológica dos gêneros, na figura da "nova mulher independente" que controla sua fecundidade, trabalha fora e tem seu próprio dinheiro(19).

Os dados do censo de 2010 apontam um declínio da taxa de fecundidade e um aumento no nível de fecundidade nos grupos de mulheres acima de 30 anos de idade. O número médio de filhos nascidos vivos por mulher ao final de seu período fértil, no Brasil, foi de 1,86 filho em 2010, bem inferior ao do Censo 2000, 2,38 filhos $^{(20)}$.

Observou-se também que crianças nascidas por parto cesáreo obtiveram uma melhor classificação do peso. Aquelas que frequentavam creches localizadas em setores mais nobres da cidade de Goiânia, onde as mães possuíam trabalho remunerado e um maior nível de escolaridade a relação peso/estatura também foi maior.

De acordo com outra pesquisa, as crianças que apresentaram um índice peso/idade aos seis meses menor ou igual a menos dois desvios-padrão da referência, tiveram um risco 10 vezes maior de suspeita de atraso no desenvolvimento(14). O que se assemelha com o encontrado nas avaliações do presente estudo, onde as crianças com menor comprimento possuíam maior risco de atraso na linguagem. Já as que tiveram maior Apgar no $5^{\circ}$ minuto obtiveram menor risco de atraso na linguagem e no desenvolvimento de uma forma geral.

Em estudo realizado com crianças de seis a 70 meses de idade em Catanduva/SP, ao analisar concomitantemente os resultados globais do Teste de Denver II e o estado nutricional, não se constatou diferenças significantes na prevalência de $\operatorname{casos}^{(12)}$.

O estudo do desenvolvimento infantil passa pela compreensão das múltiplas conexões na qual a criança está inserida e são inúmeros os fatores associados ao atraso no desenvolvimento. Рara a implementação de programas de promoção da saúde, é preciso considerar a complexidade e a dinâmica das políticas públicas que estão relacionadas às oportunidades e às condições socioeconômicas e culturais no desenvolvimento das crianças $^{(16)}$.

\section{CONCLUSÃO}

Com base nas análises o estudo verificou diferença estatisticamente significativa de acordo com o sexo nos seguintes aspectos: as crianças de sexo feminino apresentaram menor perímetro cefálico ao nascer e maior tempo de internação pós-nascimento, estavam com maior peso e relação peso/estatura na avaliação, apresentaram menor risco de atraso no desenvolvimento pessoal-social. A maior parte das crianças com peso abaixo da linha de normalidade era de sexo masculino e a maioria das classificadas como sobrepeso era do sexo feminino.

As crianças que obtiveram uma melhor classificação do peso ou maior relação peso/estatura tiveram influência de fatores socioeconômicos direta ou indiretamente e o comprimento foi inversamente proporcional ao risco de atraso no desenvolvimento da linguagem.

Embora a maior parte das crianças apresente taxa de crescimento normal, é importante que aquelas que apresentaram risco nutricional sejam acompanhadas para a investigação de possíveis causas com atenção especial para o desmame, alimentação, cuidados com a criança e afeto, além de orientar a mãe e os cuidadores sobre a alimentação complementar adequada para a idade.

Verificou-se também, que as crianças apresentaram uma considerável prevalência de risco para problemas no desenvolvimento, embora este valor possa ter sido influenciado pelo risco detectado na área da linguagem. Com esses achados, reforça-se a necessidade da estimulação para desenvolvimento da linguagem, tanto no ambiente familiar quanto dentro das creches, pois durante esse período, a criança adquire conhecimentos que serão fundamentais na vida adulta. É importante que se promovam estratégias de prevenção de possíveis problemas no desenvolvimento dessas crianças juntamente com os pais e cuidadores da creche. 
Рara futuras pesquisas, sugere-se que seja realizada comparação entre crianças de classes socioeconômicas mais elevadas e/ou frequentadoras de creches privadas para testar a influência do nível social e ambiental no crescimento e desenvolvimento infantil.

\section{REFERÊNCIAS}

1. Ministério da Saúde. Acompanhamento do Crescimento e Desenvolvimento Infantil. Brasília (Brasil). Série Cadernos de Atenção Básica. Normas e Manuais Técnicos. 2002;(11. Série A). 2. Carvalho MF, Lira PIC, Romani SAM, Santos IS, Veras A A C A, Batista Filho M. Acompanhamento do crescimento em crianças menores de um ano: situação nos serviços de saúde em Pernambuco, Brasil. Cad. Saúde Pública. 2008;24(3):675-85. 3. Torres AAL, Furumoto RAV, Alves ED. Avaliação Antropométrica de pré-escolares - comparação entre os referenciais: NCHS 2000 e OMS 2005. Rev. Eletr. Enf. [Internet] 2007 [cited 2012 mar 30];9(1):166-75. Available from:

http://www.fen.ufg.br/revista/v9/n1/v9n1a13.htm.

4. Lordelo ER, Chalhub AA, Guirra RC, Carvalho CS. Contexto e desenvolvimento cognitivo: freqüência à creche e evolução do desenvolvimento mental. Psicol Refl Crít. 2007;20(2):324-34. 5. Gioda FR, Ribeiro CM. Aquisição e refinamento do sentar independente: um estudo transversal. Rev. Bras. Cineantropom. Desempenho Hum. 2006;8(4):91-8

6. Sabates A, Mendes L. Perfil do crescimento e desenvolvimento de crianças entre 12 e 36 meses de idade que freqüentam uma creche municipal da cidade de Guarulhos. Ciênc Cuid Saúde. 2007;6(2):16470

7. Andrade SA, Santos DN, Bastos AC, Pedromônico MRM, AlmeidaFilho N, Barreto ML. Ambiente familiar e desenvolvimento cognitivo infantil: uma abordagem epidemiológica. Rev. Saúde Pública. 2005;39(4):606-11.

8. Eickmann SH, Maciel AMS, Lira PIC, Lima MC. Fatores associados ao desenvolvimento mental e motor de crianças de quatro creches públicas de Recife, Brasil. Rev. Paul. Pediatr. 2009;27(3):282-88. 9. Wanderlind F, Martins GDF, Hansen J, Macarini SM, Vieira ML. Diferenças de gênero no brincar de crianças pré-escolares e escolares na brinquedoteca. Paidéia (Ribeirão Preto) 2006;16(34):263-73.

10. Souza SC, Leone C, Takano OK, Moratelli HB. Desenvolvimento de pré-escolares na educação infantil em Cuiabá, Mato Grosso, Brasil. Cad. Saúde Pública 2008;24:1917-26.

11. Ministério da Saúde: Fundamentos Técnico-Científicos e Orientações Práticas para o Acompanhamento do Crescimento e Desenvolvimento. $1^{\text {a }}$ edição, Brasília; 2001.

12. Biscegli TS, Polis LB, Santos LM, Vicentin M. Avaliação do estado nutricional e do desenvolvimento neuropsicomotor em crianças freqüentadoras de creche. Rev. Paul Pediatr. 2007;25:337-42. 13. Associação Brasileira de Empresas de Pesquisa. Critério Padrão de Classificação Econômica Brasil [Internet]. São Paulo: ABEP; 2008 [cited 2012 mar 30]. Available from:

http://www.abep.org/novo/FileGenerate.ashx?id=257.

14. Halpern R, Giugliani ERJ, Victora CG, Barros FC, Horta BL. Fatores de risco para suspeita de atraso no desenvolvimento neuropsicomotor aos 12 meses de vida. J Pediatr. 2000;76(6):421-8. 15. Cachapuz RF, Halpern R. A influência das variáveis ambientais no desenvolvimento da linguagem em uma amostra de crianças. Revista da AMRIGS. 2006;50(4):292-301.

16. Pilz EML, Schermann LB. Determinantes biológicos e ambientais no desenvolvimento neuropsicomotor em uma amostra de crianças de Canoas/RS. Ciênc. saúde coletiva. 2007;12(1):181-90.

17. Cipollone L. Diferença sexual, dimensão interpessoal e afetividade nos contextos educacionais para a infâncial. ProPosições. 2003;14(3):25-39.

18. Nobre FSS, Costa CLA, Oliveira DL, Cabral DA, Nobre GC, Caçola P. Análise das oportunidades para o desenvolvimento motor. Rev. Bras. Crescimento Desenvolvimento Hum. 2009;19(1):9-18. 19. Giffin K. Pobreza, desigualdade e eqüidade em saúde: considerações a partir de uma perspectiva de gênero transversal. Cad. Saúde Pública. 2006;16(34)263-73.
20. Instituto Brasileiro de Geografia e Estatística [Internet]. Brasília: Comunicação Social. Censo 2010: País tem declínio de fecundidade e migração e aumentos na escolarização, ocupação e posse de bens duráveis. [cited 2011 nov 16] Notícias do Censo 2010. Available from:

http://www.ibge.gov.br/home/presidencia/noticias/noticia visualiza .php?id noticia=2018\&id pagina=1.

Artigo recebido em 08.07.2010.

Aprovado para publicação em 13.03.2012.

Artigo publicado em 30.03.2012. 